\title{
Tamoxifen non-estrogen receptor mediated molecular targets
}

\author{
Tatiana Bogush, Evgeny Dudko, Elena Bogush, Boris Polotsky, Sergei Tjulandin, Mikhail \\ Davydov
}

\author{
N.N. Blokhin Russian Cancer Research Center under the Russian Academy of Medical Sciences, \\ Russian Federation, Moscow
}

\begin{abstract}
Recent experimental studies revealing new biological effects of tamoxifen on tumor cells both expressing and not expressing different types of estrogen receptors (ER $\alpha$ and ER $\beta$ ) show new aspects of a seemingly well known agent. This review describes tamoxifen targets, the blocking of which leads to inhibition of tumor cell growth and angiogenesis, stimulation of programmed cell death (apoptosis, autophagia and necrosis), inhibition of multidrug resistance, invasion and metastasis. Since outcomes of tamoxifen action on cells are prognostically good from the point of view of both tumor growth/metastasis inhibition and tumor response to drug therapy, the authors believe this is an extremely important addition to tamoxifen antiestrogenic effect. Arguments are provided to consider the strategy of long-term tamoxifen treatment proposed by Professor Craig V. Jordan in the 1970 s that is also applicable to the treatment of other tumors. This is, first of all, the fact that expression of estrogen receptor-beta that can also be targeted by tamoxifen therapy in solid tumors of practically all known sites and histologies. The authors believe that molecular biological screening of patients with respect to expression of tamoxifen cellular targets other than ER $\alpha$ and ER $\beta$ is needed to use to the full all tamoxifen biological activities other than modulation of estrogen receptors during long-term adjuvant therapy for cancers of various sites.
\end{abstract}

Correspondence: Tatiana Bogush, Head of Medical Chemistry Laboratory, Kashirskoje shosse 24, 115478, Moscow, Russian Federation.

Tel. +7.499.324.18.64. E-mail: bogush@orc.ru

Key words: tamoxifen, apoptosis, angiogenesis, metastasis, multidrug resistance.

Contributions: the authors contributed equally.

Conflict of interests: the authors declare no potential conflict of interests.

Funding: the study was supported by Russian Foundation for Basic Research (Grants 10-04-00551-a and 12-04-00028-a).

Received for publication: 22 March 2012.

Revision received: 18 June 2012.

Accepted for publication: 5 July 2012.

This work is licensed under a Creative Commons Attribution NonCommercial 3.0 License (CC BY-NC 3.0).

CCopyright T. Bogush et al., 2012

Licensee PAGEPress, Italy

Oncology Reviews 2012; 6:e15

doi:10.4081/oncol.2012.e15

\section{Introduction}

Notwithstanding the rapid progress in hormonal anticancer therapies and resulting new effective agents, tamoxifen has been for many, many years the most outstanding and invariably effective cancer therapy. This agent is considered a gold standard in the treatment of estrogen receptor (ER)-positive breast cancer. This is the first and the oldest targeted drug, remaining a leader in breast cancer treatment. ${ }^{1,2}$

Estrogen receptors are the most important cell target in view of both control of carcinogenesis and inhibition of tumor cell growth. Results of studies over many years (since the early 1970s) of tamoxifen treatment of patients with ER-positive breast cancer are an excellent confirmation of this finding. The drug is also effective as adjuvant therapy and considerably improves follow-up outcomes of surgery by reducing the risk of disease recurrence and death. Tamoxifen antitumor effect is first of all due to the ability to selectively block the ERs found in most breast cancer patients. This explains the continuing interest in antiestrogen therapy for breast cancer.

The situation started to change gradually in 1996 when new ER-type estrogen receptors beta (ER $\beta$ ) were discovered. Since then, the ERs, whose status was determined in breast tumors, were referred to as estrogen receptors alpha (ER $\alpha)$. As far as ER $\beta$ is concerned, they have been found in a variety of tumor sites, including carcinomas of the colon, esophagus, stomach, lung, brain, prostate, pancreas. ${ }^{3,4}$ This provided a rationale for broadening indications of tamoxifen in ER $\alpha$-negative ER $\beta$-positive tumors, including breast cancer and tumors of other sites. Furthermore, recent experimental studies have revealed new biological effects of tamoxifen on tumor cells. A large spectrum of tamoxifen targets other than estrogen receptors have been discovered that are key mediators of signal pathways activating cell proliferation, determining aggressive course of neoplastic disease or tumor sensitivity to chemotherapy. As a result, new aspects of a seemingly well known agent have become apparent, as well as prospects for its new clinical indications, in particular in the treatment of solid tumors other than breast cancer. This includes its use in combinations with current target therapies. These new targets are analyzed below.

\section{Inhibition of protein kinase C}

Protein kinase C (PKC) is serin/threonin-specific protein kinase expressed on practically all mammalian cells. It plays an extremely important role in intracellular signaling. PKC substrates in various cell types include nuclear proteins, cytoskeletal proteins, enzymes. PKC mediates transmission of a broad range of external signals including those regulating cell growth.

Tamoxifen produced cytotoxic effect on human prostate cancer cell cultures PC3 and PC3-M that at a molecular level was associated with 
inhibition of PKC which was followed by induction of p21(wafl/cip1), $\mathrm{Rb}$ dephosphorylation and $\mathrm{G}_{1} / \mathrm{S}$ phase cell arrest. Interestingly, Ro318220, a PKC specific inhibitor, produced a similar effect. ${ }^{5}$ Tamoxifen demonstrated similar effects on cells of other tumor types, such as hepatocellular carcinoma ${ }^{6}$ and astrocytoma. ${ }^{7}$ Tamoxifen inhibiting effect on PKC was also demonstrated experimentally on malignant gliomas. ${ }^{8}$ As shown on breast cancer MCF-7 cell culture, tamoxifen exerts its antiproliferative activity through direct interaction with PKCepsilon associated with tumor cell differentiation and growth. ${ }^{9}$

\section{Suppression of metastasis}

Several authors described an important effect of tamoxifen, i.e. antimetastatic activity that might take place regardless of ER expression on human cells and was associated with impairment of many cellular targets. For example, tamoxifen up-regulated expression of tissue inhibitor of metalloproteinases-1, TIMP-1, and down-regulated expression of metalloproteinase 9 in lung adenocarcinoma SPC-A-1 and breast cancer MCF-7 cells, that led to inhibition of cell invasion in matrigel. Both of these cell lines were ER-positive. ${ }^{10}$ Tamoxifen antimetastatic activity was described on ER-positive colonic cancer cells with high metastatic potential. The antiestrogen down-regulated expression of matrix metalloproteinase MMP7 and inhibited cell migration into the wound in monolayer culture. ${ }^{11}$ Inhibition of cell growth and migration into the wound was also seen on cultures of ER-negative human thyroid cancer cells. This effect was confirmed in vivo on FTC133 cell xenografts in nude mice. Tamoxifen inhibited the growth of FTC133 xenografts in nude mice. ${ }^{12}$ In murine melanoma B16BL6 cell culture, tamoxifen inhibited mRNA expression and protein activities of matrix metalloproteases. Experiments in vivo on a murine model demonstrated a significant inhibition of melanoma metastasis into lungs. ${ }^{13}$ In rat breast cancer wild-type Mat B-III cells and with overexpression of urokinase receptor uPAR tamoxifen inhibited UPAR gene transcription, mRNA expression and protein production. In in vivo experiments the antiestrogen both reduced tumor size and inhibited metastasis after tumor cell transplantation to Fisher rats. ${ }^{14}$

\section{Activation of programmed cell death}

The tamoxifen ability to enhance programmed cell death or apoptosis contributes largely to its cytostatic effects.

A variety of non-ER mediated apoptosis enhancing mechanisms in cells of different histogenesis are described, including inhibition of phospholipase C-, D- and protein kinase C-mediated pathways, that is a possible mechanism of action independent of ER expression. ${ }^{15}$

Tamoxifen effect on ER-positive breast cancer cells leads to activation of caspases 6,7 and $9 .{ }^{16}$ Tamoxifen induced apoptosis of rat glioma C6 cells via inhibition of AKT activation and JNK transitory activation, whereas MAP kinase ERK evidenced sustained activation in response to the drug treatment. ${ }^{17}$

A mechanism is described for apoptosis stimulation under the tamoxifen effect on cells over-expressing antiapoptotic proteins. Tamoxifen treatment of Bcl-2 over-expressing clones was found to induce apoptosis via activation of c-Jun N-terminal kinase (JNK), p38 kinase and phosphorylation of c-Jun, in parallel with increasing LNAbindinig activity of AP-1, expression of FasL and activating caspase 8. No such effects were seen in cells under-expressing this protein. ${ }^{18}$ Tamoxifen was also shown to induce apoptotic changes in cells both by up-regulating ${ }^{15,19}$ and keeping stable levels of Bcl-2 $2^{20,21}$ in cells.
From the point of view of variability of effects on cells, the influence of tamoxifen on expression of other proteins involved in apoptotic processes is of interest. Similarly to the effects on Bcl-2, tamoxifen may produce variable effects on levels of these proteins. Tamoxifen inhibited growth and induced apoptosis in cells of human bile duct carcinoma QBC939, up-regulated expression of p53 and p21(wafl/cip1), and induced $\mathrm{G}_{0}-\mathrm{G}_{1}$ phase cell cycle arrest. Downregulation of cyclin D and $\mathrm{C}$ Myc expression, upregulation of Bcl-2, Bax and caspases 6, 7 and 9 were also observed in tamoxifen-treated cells. ${ }^{22}$

Tamoxifen can activate mitochondria-dependent apoptosis. ${ }^{23}$ Tamoxifen enhanced apoptosis and oxidative stress via mitochondriadependent and nitric oxide (NO)-dependent pathways associated with increased intramitochondrial $\mathrm{Ca}^{2+}$ concentration. Tamoxifen stimulation of mitochondrial NO synthase resulted in suppression of mitochondrial respiration, reduced release of cytochrome $\mathrm{C}$, increased mitochondrial lipid peroxidation, and decreased aggregation of mitochondria. ${ }^{24,25}$ All these processes finally led to tumor cell apoptosis with formation and phagocytosis of apoptotic bodies.

Type II programmed cell death is associated with autophagia program, i.e. degradation of organelles and cytoplasmic material with intracellular structures contributing to this process. Treatment with various selective modulators of ERs (including tamoxifen) led to inhibition of type II cell death in a dose-dependent manner. ${ }^{26}$ Further studies discovered that autophagia activation by tamoxifen began with depolymerization of actin and degradation of intermediate filaments. ${ }^{27}$ Tamoxifen-induced activation of autophagia was confirmed by a series of studies on cell lines from primary and metastatic carcinomas of the breast, ${ }^{28}$ colon $^{29}$ and lymphoma. ${ }^{30}$

Finally, tamoxifen influences type III cell death, i.e. programmed necrosis. The necrosis may be induced by activating apoptosis via binding of ligands such as FasL (a member of tumor necrosis factor family) and TRAIL (TNF-related apoptosis-inducing ligand), and by inducing overexpression of protoapoptotic protein Bax simultaneously, or by inhibiting activity of caspases, or by inducing overexpression of antiapoptotic proteins. Tamoxifen induces apoptosis in Fas receptor-positive cells through regulation of expression of Fas ligand. This mechanism explains selective stimulation of apoptosis in osteoclasts that prevents bone resorption and osteoporosis. ${ }^{31}$

What cell cycle phases are associated with different types of cell death? Unlike apoptosis that can start in various cell cycle phases, including mitosis per se as a mitotic catastrophe, autophagic death is observed mainly in non-proliferating cells. However, if apoptotic mechanisms are inhibited in proliferating cells, e.g. through caspase inactivation, then death of the proliferating cells is by a mechanism of programmed necrosis. Combinations of types of tamoxifen-induced cell death present multiple targets for this antiestrogen.

Interesting findings were reported by a study of tamoxifen effect on expression of 36 proteins in MCF7 breast cancer cells. ${ }^{32}$ Tamoxifen was shown to change expression of heat shock proteins, various structural proteins, and proteins involved in apoptosis, posttranslational modifications, glycolysis, RNA processing, cell cycle progression, DNA transcription and translation, cell differentiation. A total of 9 proteins from different groups appeared up-regulated, while 7 others were down-regulated. The authors also demonstrated that tamoxifen altered functioning of E6-associated protein (E6AP, also UBE3A) which, on the one hand, was a coactivator of estrogen receptor alpha and, on the other hand, was a E3 ubiquitin-protein ligase involved in degradation of various proteins. After binding to estrogen receptors alpha, tamoxifen interfered with E6AP coactivation function followed by E6AP transfer from the nucleus into cytoplasmatic space and degradation. Biological effects in this case include $\mathbf{G}_{0}-\mathbf{G}_{1}$ growth arrest and appearance of cells in apoptotic state.

Tamoxifen was found to produce synergic effects if used with other apoptosis activators which might be of great importance for counteract- 
ing drug resistance. For instance, co-treatment with tamoxifen and TRAIL down-regulates antiapoptotic proteins FLIP and Bcl-2, while simultaneously up-regulating proapoptotic proteins FADD, tBid, Bax, caspases 8 and 9 in breast tumors, regardless of their ER status. ${ }^{33}$

The augmentation of tamoxifen apoptotic effects was observed when the antiestrogen was used in combination with apoptosis inducer, roscovitine, that inhibited cyclin-dependent kinases mainly through effects on kinases CDK2, CDK7 and CDK9. Co-treatment with the two agents was associated with synergism of apoptotic effect in ER-positive breast cancer cells and antagonism in ER-negative cells. ${ }^{34-36}$

Therefore, stimulation of apoptosis, autophagia or necrosis depending on cell context contributes to tamoxifen effects and broadens the range of indications for tamoxifen administration, including its use in combination with other agents.
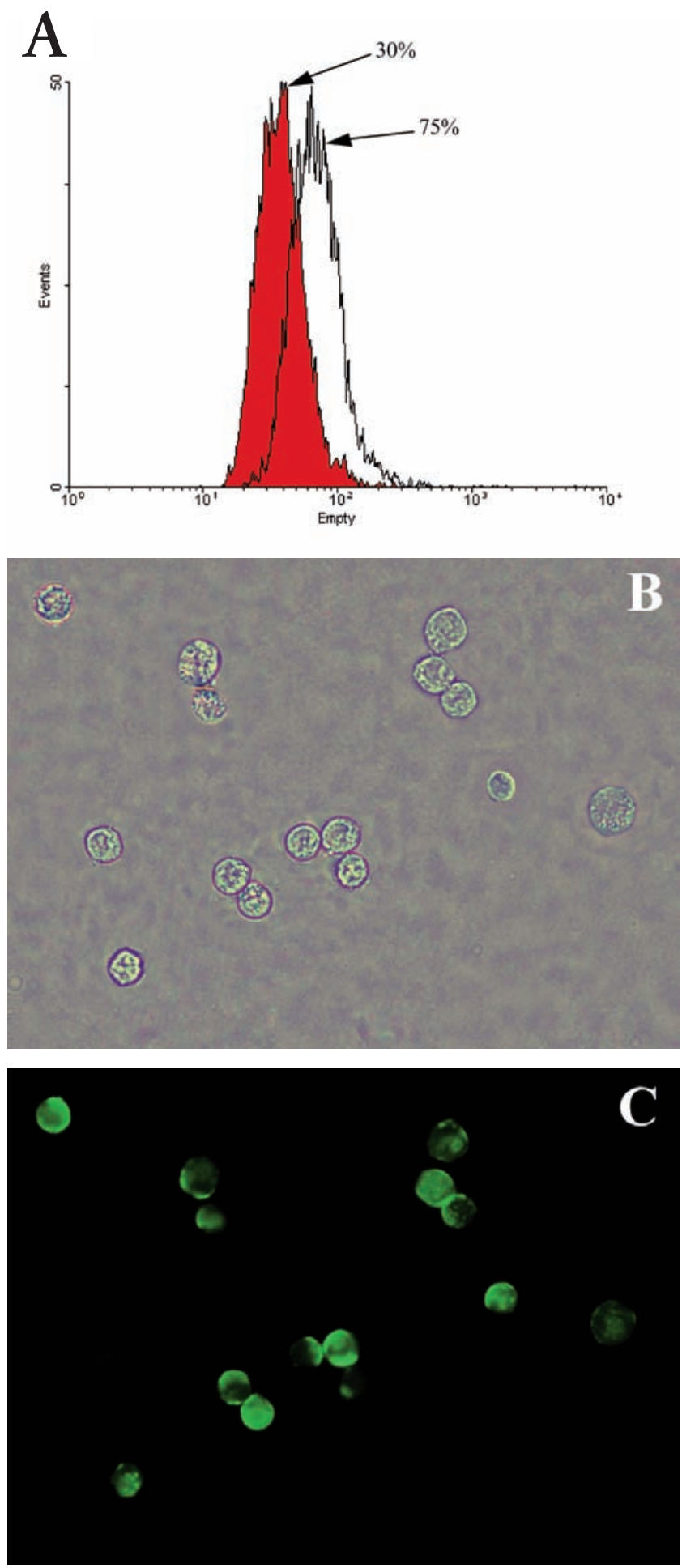
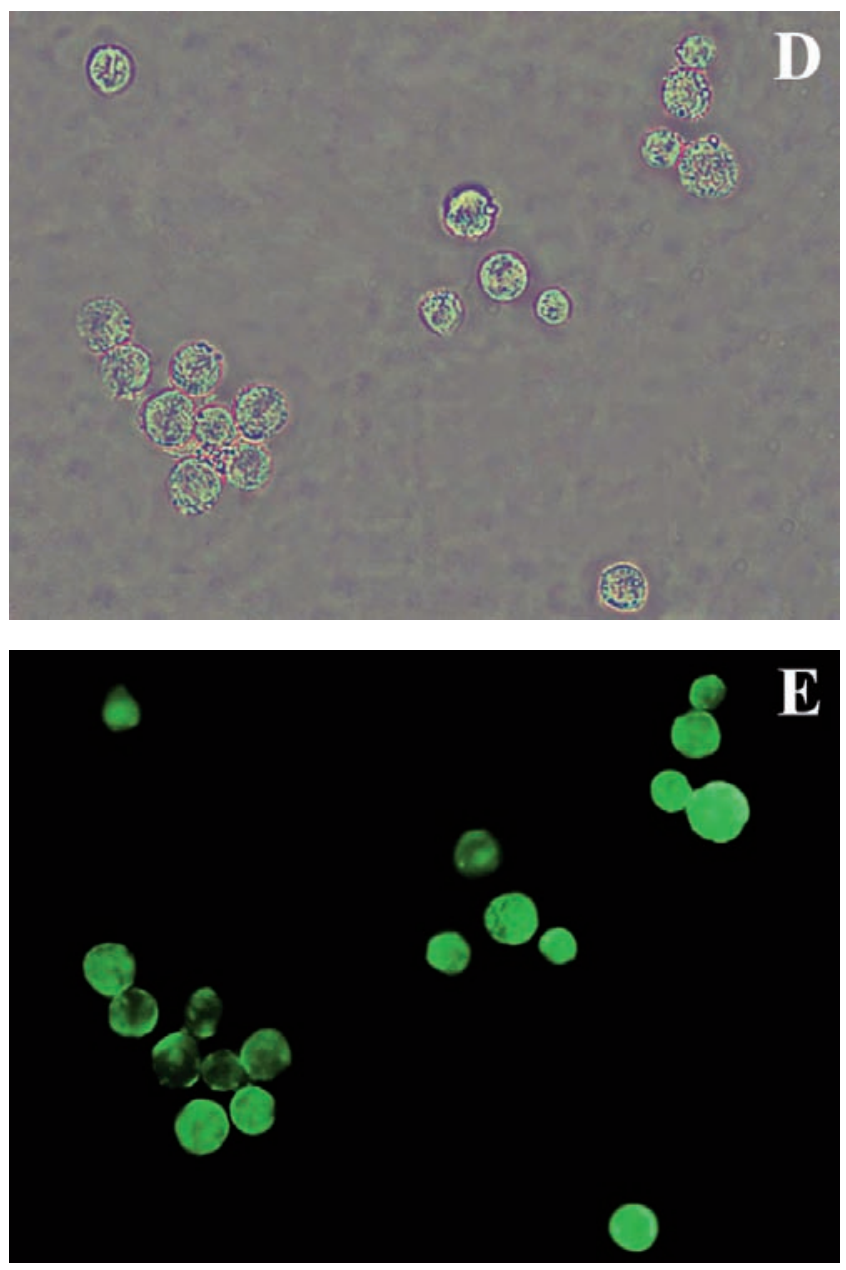

Figure 1. Tamoxifen effects on specific interaction of monoclonal antibody (clone 4E3, Abcam) with P-glycoprotein (Pgp) in Jurkat human T-lymphoblastic leukemia cell line. (A) Cell distribution with respect to fluorescence intensity (flow cytometer BD FACSCanto II), $\mathrm{x}$ axis: fluorescence intensity (conditional units); $y$ axis: the number of cells. Shaded bars show cell fluorescence intensity without tamoxifen treatment; blank bars show cell fluores-cence intensity after incubation with tamoxifen. Figures correspond to percentages of specifically fluorescent cells with respect to isotype control. (B-E) Cell photographs after fluorescent staining with anti-Pgp monoclonal antibody made using a light fluorescent microscope Leica DMI 6000B (magnification $400 x)$. Cells in the light and fluorescent fields: (B) and (C) free from tamoxifen treatment. (D) and (E) after tamoxifen treatment. 


\section{Inhibition of angiogenesis}

It is currently commonly understood that angiogenesis is a necessary condition for malignant growth and metastasis. There is a vast literature on tamoxifen antiangiogenic properties similar to those of other antiangiogenic agents. ${ }^{37-39}$

The tamoxifen ability to inhibit angiogenesis has been tested on classical models of antiangiogenic studies, such as measurement of microvascular density in rat fibrosarcoma, aortic ring assay, chicken chorioallantoic membrane assay and rabbit corneal pocket assays. ${ }^{39}$
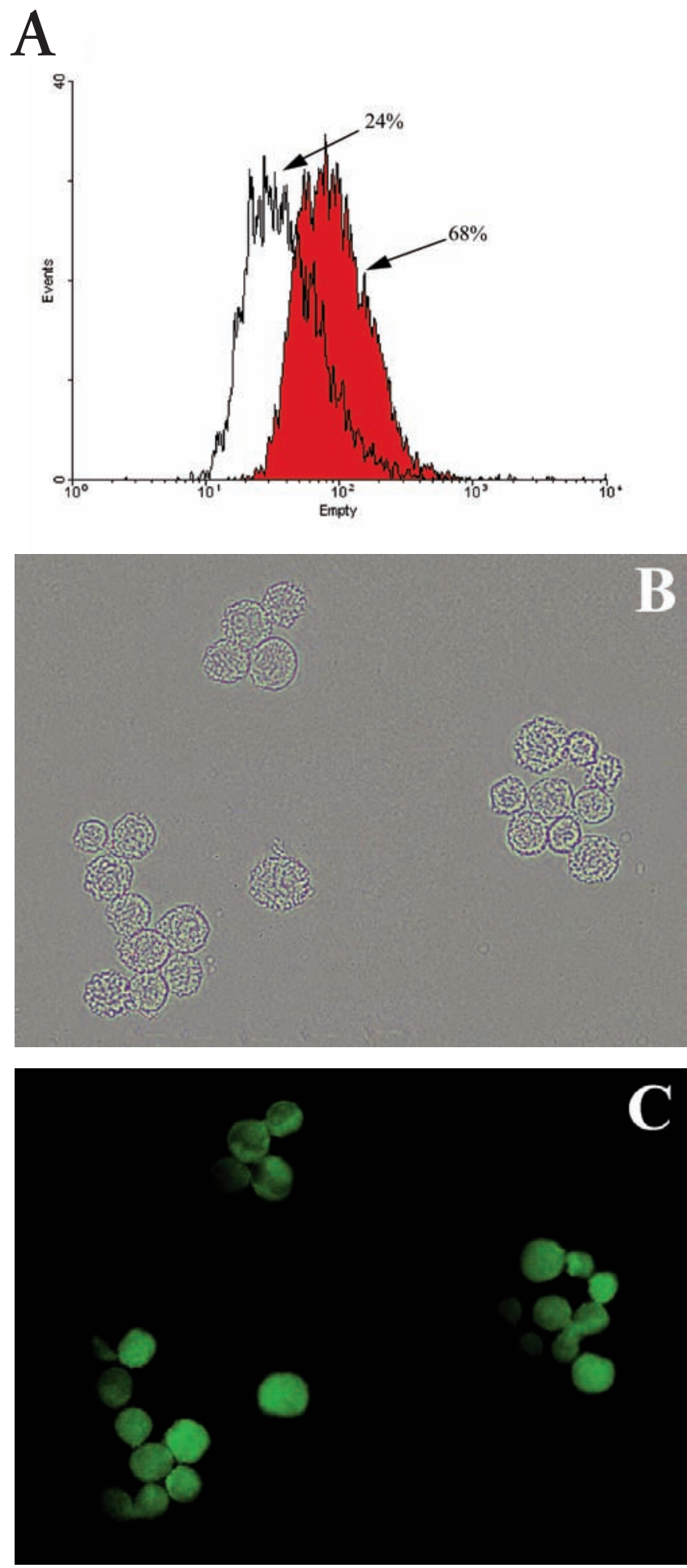

Tamoxifen angiogenesis inhibition effects were extensively studied both in vitro and in vivo, and were seen on both ER-positive and ERnegative tumor models. ${ }^{40,41}$

There are several mechanisms of tamoxifen antiangiogenic effect, such as inhibition of cyclin-dependent endothelial cell growth, ${ }^{42}$ modulation of transforming growth factor beta (TGF- $\beta$ ) in breast cancer cells, ${ }^{43}$ inhibition of vascular endothelial growth factor (VEGF) and basic fibroblast growth factor (bFGF). ${ }^{40,44-46}$ VEGF is a key promoter of tumor angiogenesis. VEGF expression rises in response to hypoxia, activation of oncogenes and various cytokines. VEGF induces endothelial cell proliferation and migration, and inhibits apoptosis. The antian-
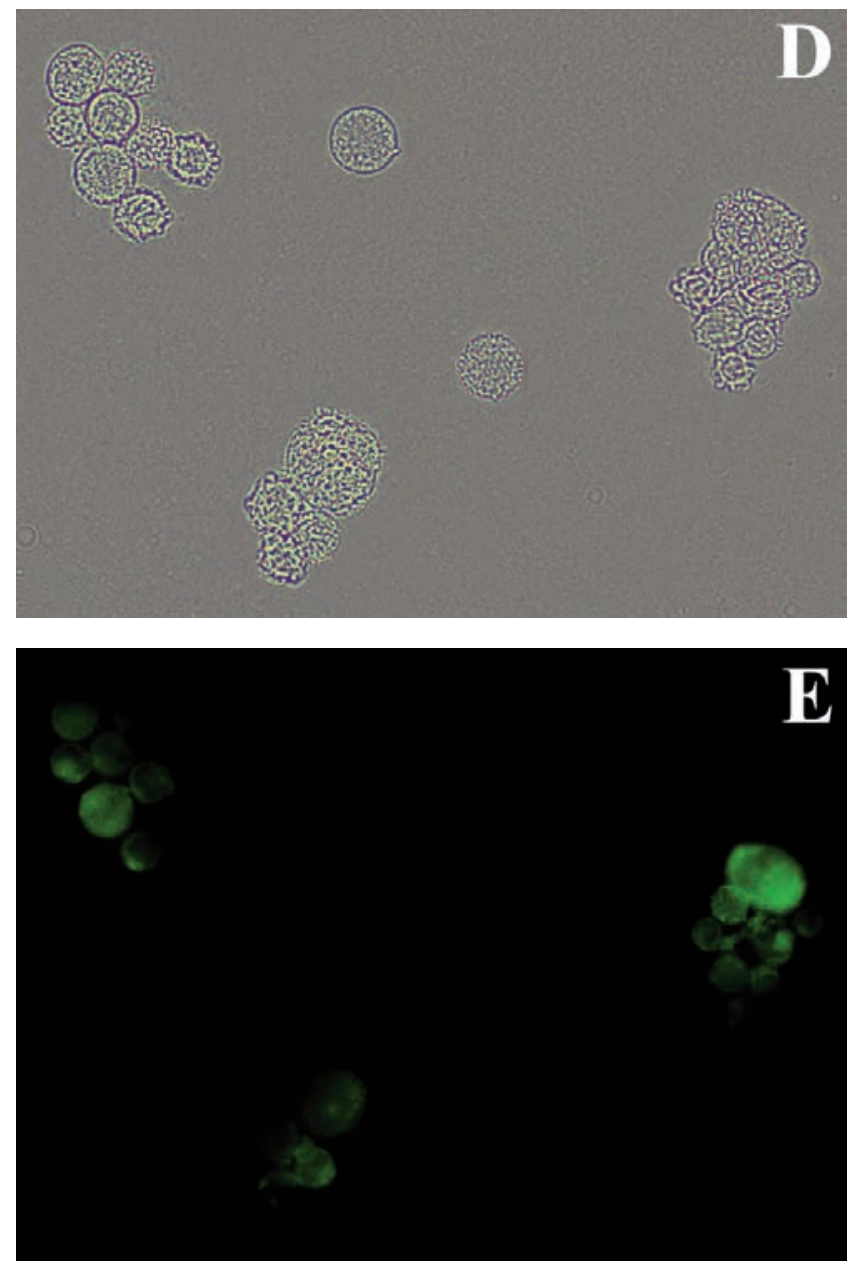

Figure 2. Tamoxifen effects on specific interaction of monoclonal antibody (clone MRPm5, Abcam) with MRP1 in HeLa human cervical cancer cell line. (A) Cell distribution with respect to fluorescence intensity (flow cytometer BD FACSCanto II). $x$ axis: fluorescence intensity (conditional units); $y$ axis: the number of cells. Shaded bars show cell fluorescence intensity without tamoxifen treatment; blank bars show cell fluorescence intensity after incubation with tamoxifen. Figures correspond to percentages of specifically fluorescent cells with respect to isotype control. (B-E) Cell photographs after fluorescent staining with anti-MRP1 monoclonal antibody made using a light fluorescent microscope Leica DMI 6000B (magnification 400x). Cells in the light and fluorescent fields: (B) and (C) free from tamoxifen treatment. (D) and $(\mathrm{E})$ after tamoxifen treatment. 
giogenic activity of tamoxifen and other antiestrogen agents (nafoxidine, clomifen and ICI 182,780) is not associated with tumor ER status and is due to direct inhibition of VEGF and bFGF, as shown in a 6-day chicken chorioallantoic membrane assay. Tamoxifen is also shown to inhibit VEGF secretion in ER-positive human breast cancer cell line MCF-7. ${ }^{44}$ Furthermore, tamoxifen decreased the levels of proangiogenic VEGF and angiogenin while increasing antiangiogenic angiostatin levels in the normal human breast tissue. ${ }^{45}$ Tamoxifen inhibitory effect on angiogenesis is also associated with increased expression of matrix metalloproteinases MMP2, MMP9 and antiangiogenic protein
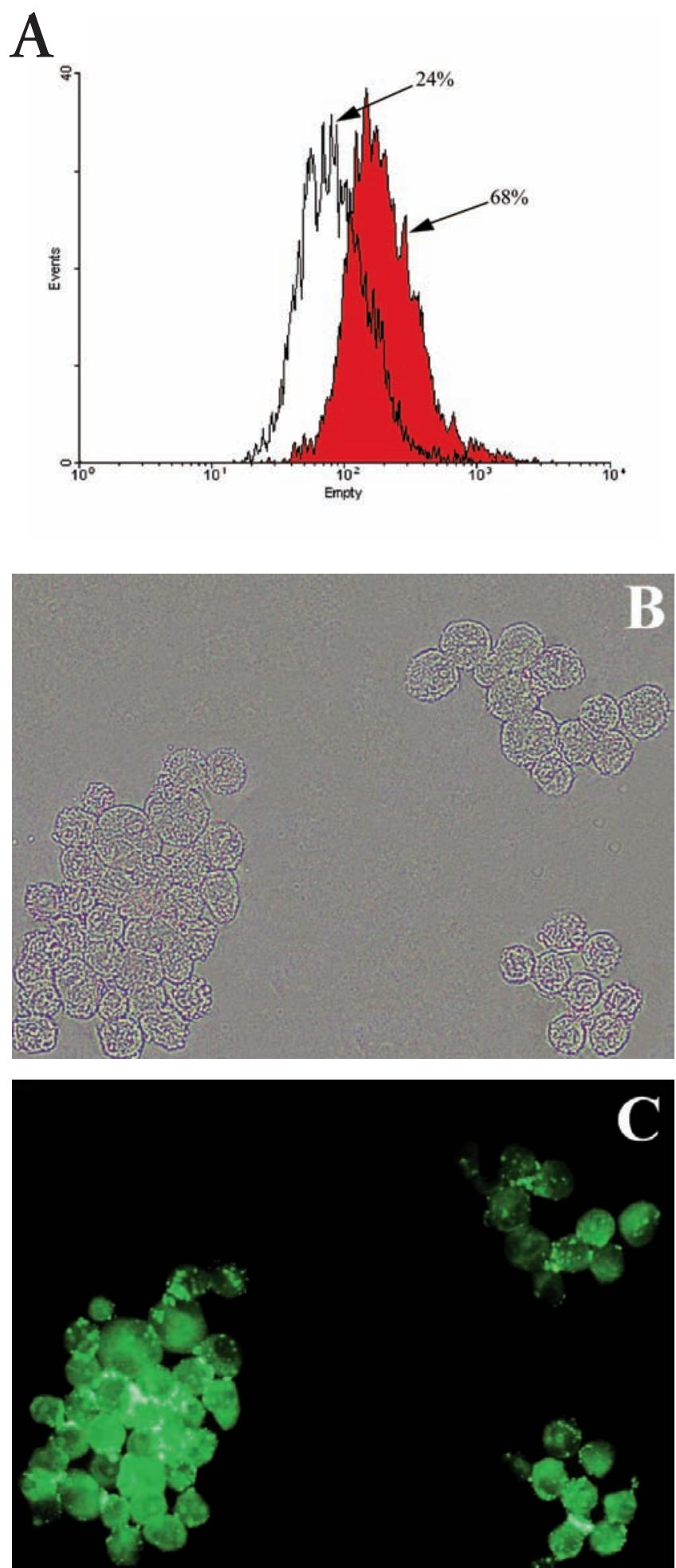

endostatin. ${ }^{47}$ Effect of tamoxifen in combination with estradiol on xenografts of breast cancer MCF7 in nude mice led to elevation in levels of endostatin and MMP2, and MMP2 and MMP9 activity. Tamoxifen produced the same effect, i.e. increased expression of the above-mentioned proteins, on MCF7 cells in vitro in the absence of estradiol. Tamoxifen effect on MCF7 cells was also associated with increased expression of tissue inhibitors of metalloproteinases, TIMP-1 and TIMP-2. ${ }^{48}$ Another mechanism of tamoxifen antiangiogenic effects is upregulation of interleukin receptor antagonist-1 (IL-1Ra) that interferes with IL-1 $\alpha$ and IL-1 $\beta$ signal transmission. Since both IL-1 $\alpha$ and
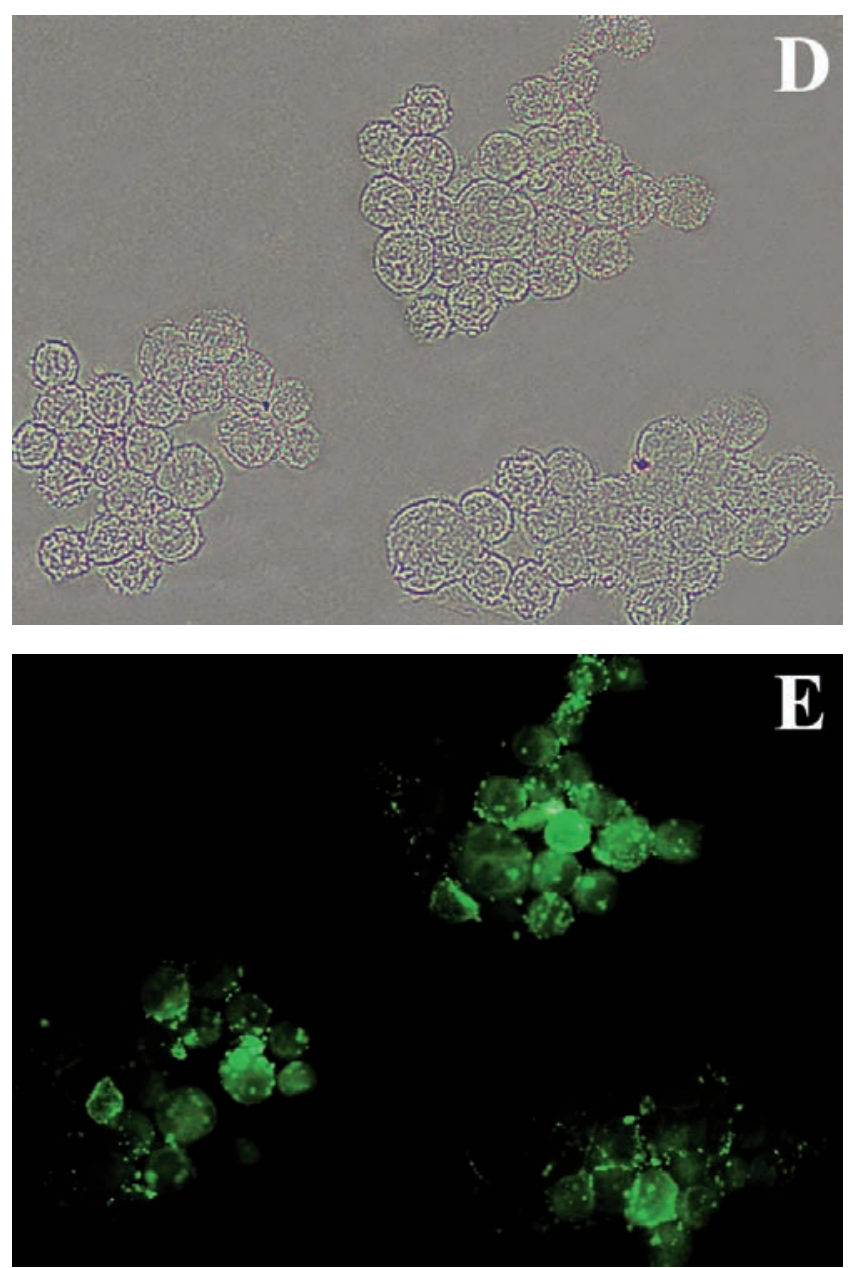

Figure 3. Tamoxifen effects on specific interaction of monoclonal antibody (clone LMR5, Abcam) with lung resistance-related protein (LRP) in A549 human lung adenocarcinoma cell line. (A) Cell distribution with respect to fluorescence intensity (flow cytometer BD FACSCanto II). $\mathrm{x}$ axis: fluorescence intensity (conditional units); y axis: the number of cells. Shaded bars show cell fluorescence intensity without tamoxifen treatment; blank bars show cell fluorescence intensity after incubation with tamoxifen. Figures correspond to percentages of specifically fluorescent cells with respect to isotype control. (B-E) Cell photographs after fluorescent staining with anti-LRP monoclonal antibody made using a light fluorescent microscope Leica DMI 6000B (magnification $\times 400)$. Cells in the light and fluorescent fields: (B) and (C) free from tamoxifen treatment. (D) and (E) after tamoxifen treatment. (C) and (E) clearly show granularity of the staining with monoclonal antibody against lung resistance-related protein that, unlike other multidrug resistance proteins, is found within ribonucleoprotein particles or vaults rather than in a free state. These vaults can form tubular structures that are seen as fluorescent granularity during fluorescent analysis. 
IL-1 $\beta$ promote tumor angiogenesis, inhibition of these interleukins decreases activation of neoangiogenesis. Tamoxifen increased IL-1Ra expression in a murine model of human breast cancer and thus inhibited angiogenesis. ${ }^{49}$ Heparin-binding polypeptide, angiogenin, is the most important and a most powerful angiogenic factor. It binds to the endothelial cell surface and induces cell proliferation, ${ }^{50}$ activates endothelial cell proteases, ${ }^{51}$ and promotes tube formation by endothelial cells. ${ }^{52}$ Elevated angiogenin levels were found in tumors of various sites. ${ }^{33-60}$ Angiogenin levels were increasing in the series normal breast tissue $\rightarrow$ carcinoma in situ $\rightarrow$ invasive carcinoma and demonstrated positive correlation with high tumor grade, positive estrogen receptor status, and hypoxia-inducible factor. ${ }^{61}$ Interesting findings were recently reported concerning angiogenin regulation by estradiol and tamoxifen in breast tissue. ${ }^{62}$ Extracellular angiogenin was shown to correlate significantly with estradiol in normal breast tissue in vivo, and exposure of normal breast tissue biopsies to estradiol stimulated angiogenin secretion. In breast cancer patients, angiogenin levels are significantly higher in tumors compared with the adjacent normal breast tissue. In estrogen receptor-positive breast cancer cells, estradiol increases angiogenin secretion and endothelial cell proliferation. Tamoxifen reverses both of these estradiol effects and decreases in vivo the level of angiogenin and angiogenesis. Anti-angiogenin antibody therapy decreases growth of MCF7 tumors in nude mice. Taken together, these results suggest previously unknown mechanisms of angiogenesis regulation by estrogen and the antiestrogen.

\section{Interaction with multidrug resistance proteins}

Another tamoxifen target are proteins that throw out antitumor agents with different structure and mechanism of action from cells and are associated with multidrug resistance mechanism (MDR). These are transport proteins, members of the $\mathrm{ABC}$ transporter family, such as P-glycoprotein (Pgp), multidrug resistance-associated proteins (MRP) and breast cancer resistance protein. The group also includes multidrug resistance marker major vault protein, also refereed to as lung resistance-related protein (LRP).

Tamoxifen enhanced specific activity of some cytostatics against many tumors with Pgp-associated MDR phenotype in cell cultures and animal models. ${ }^{63,64}$ Furthermore, tamoxifen interacted with Pgp, while some antitumor agents competed for binding to Pgp with verapamil and tamoxifen in cell cultures of different histogenesis, which might be an explanation for the above-described phenomenon. ${ }^{65,66}$ Tamoxifen was also shown to enhance effects of adriamycin, mitomycin and vindesine in human cholangiocarcinoma cell line QBC939 with MDR phenotype. However, when specific anti-Pgp antibody was added to tamoxifen combination with antitumor drugs, this antibody blocked the tamoxifeninduced downregulation of Pgp expression. ${ }^{67}$ Therefore, tamoxifen effect may be defined as recovery of response to chemotherapy in tumors with MDR phenotype due to tamoxifen influence on Pgp.

Direct experiments to investigate tamoxifen effects on monoclonal antibody-Pgp binding in human chronic myelogeneous leukemia cell line $\mathrm{K} 562$ discovered that the antiestrogen competed with the antibody for the binding. ${ }^{68} \mathrm{~A}$ similar result was obtained in a study of the effect of tamoxifen on the monoclonal antibody binding with MRP1 in human cervical carcinoma cell line HeLa. ${ }^{69}$ In our experiments (T Bogush, E Dudko, E Bogush, B Polotsky, S Tjulandin, M Davydov, unpublished data, 2012 year) tamoxifen competitive interaction with Pgp, MRP1 and LRP was visualized by fluorescent microscopy. Changes in specific fluorescent staining of cells under tamoxifen effect were confirmed by flow cytometry in all cases (Figures 1-3). Incubation with tamoxifen increased the number of specifically fluorescent cells and fluorescence intensity of some anti-Pgp antibody-stained cells in Jurkat human Tlymphoblastic leukemia cell suspension (Figure 1). On the contrary, there was a decrease in the number of specifically fluorescent cells and fluorescence intensity of some cells in response to tamoxifen treatment after HeLa and A549 lung adenocarcinoma monolayer cultures were stained with anti-MRP1 and anti-LRP monoclonal antibodies (Figures 2 and 3). These findings prove that tamoxifen interacts with MDR markers Pgp, MRP1 and LRP, and thus interferes in the interaction of antitumor drugs with these transporter proteins, consequently inhibiting the associated MDR mechanism. We believe that the tamoxifen interaction with Pgp, MRP1 and LRP should inevitably decrease the intracellular concentration of tamoxifen available for interaction with other cell targets including ER. Therefore, tamoxifen interaction with Pgp, MRP1 and LRP in MDR cells may result in a negative effect, that is a reduction in tamoxifen's own efficacy. It is obvious that the higher the overexpression of transport proteins, the greater prognostic value (with respect to tamoxifen resistance) they have. It should be emphasized, however, that this is not the case with the MDR mechanism associated with drug throw-out from cells. This is basically a new mechanism of a reduction in tamoxifen efficacy due to intracellular inactivation as a result of binding to these proteins on its way to its own targets, rather than due to Pgp, MRP1 or LRP functioning.

Table 1. Tamoxifen biological effects independent from tumor estrogen receptor status

\section{Tamoxifen biological effects \\ Stimulation of cell programmed death: apoptosis, autophagia, necrosis}

Cell proliferation inhibition

Angiogenesis inhibition

Invasion and metastasis inhibition

\section{Mechanism of tamoxifen biological effects}

Activation of caspases 6, 7,8 and 9

Activation of JNK, p38, p53, p21 and FasL

Downregulation of Bcl-2, E6AP

Inhibition of protein kinase $\mathrm{C}$

Inhibition of tranforming growth factor-betal

Inhibition of VEGF, bFGF and angiogenin

Stimulation of IL-1Ra

Activation of MMP2, MMP9 and endostatin

Stimulation of TIMP-1

Inhibition of MMP7 and 9

Inhibition of uPAR

Multidrug resistance inhibition

Interaction with multidrug resistance-associated proteins Pgp, MRP1 and LRP

VEGF, vascular endothelial growth factor; bFGF, basic fibroblast growth factor; IL-1Ra, interleukin receptor antagonist-1; MMP, matrix metalloproteinases; TIMP-1, tissue inhibitor of metalloproteinases-1; uPAR, urokinase receptor; Pgp, P-glycoprotein; MRP, multidrug resistance-associated protein; LRP, lung resistance-related protein. 


\section{Conclusions}

In conclusion, we should mention the strategy of long-term tamoxifen treatment proposed by Professor Craig V. Jordan in the 1970s that has strongly positioned tamoxifen as an inevitably effective agent in the treatment of ER-positive breast cancer for more than four decades. ${ }^{1}$ We believe that the discovery in 1996 of a new type of tamoxifen target, ER-beta, together with data on its expression in tumors of various sites, make the long-term adjuvant therapy with tamoxifen also applicable to tumors other than breast cancer. It is currently commonly understood that various ERs are expressed in tumors of all sites and histologies. Differences may be found only in frequency and intensity of expression of these tamoxifen cellular targets. ${ }^{3,4}$

Findings concerning key mediators of signal pathways and cellular receptor proteins targeted by tamoxifen, as reviewed in this paper, demonstrate that tamoxifen is a unique polyvalent targeted drug. Table 1 summarizes data of tamoxifen non-ER cellular targets. Tamoxifen effect on these targets activates or inhibits most important biological processes that control tumor growth and response to chemotherapy. As seen, outcomes of tamoxifen action on cells are prognostically good from the point of view of both tumor growth/metastasis inhibition and tumor response to drug therapy. This is an extremely important addition to tamoxifen antiestrogenic effect. We believe that molecular biological screening of patients with respect to expression of tamoxifen cellular targets other than different types of ERs is needed to use to the full all tamoxifen biological activities during long-term adjuvant cancer therapy. Without such a screening, one can hardly expect a good response to any targeted agents, and tamoxifen is no exception. This approach will help to utilize the efffect of tamoxifen in some cases with ER-negative tumors in long-term adjuvant treatment regimens. Furthermore, assessment of tamoxifen cellular targets other than estrogen receptors will help enlarge indications of adjuvant tamoxifen therapy not only in breast cancer but also in tumors in other sites.

\section{References}

1. Jordan VC. Tamoxifen: catalyst for the change to targeted therapy. Eur J Cancer 2008;441:30-8.

2. Obiorah I, Jordan VC. Progress in endocrine approaches to the treatment and prevention of breast cancer. Maturitas 2011;70:315-21.

3. Bogush TA, Dudko EA, Beme AA, et al. Estrogen receptor expression in tumors different from breast cancer. Antibiot Khimioter 2009;54:41-9.

4. Bogush TA, Dudko EA, Beme AA, et al. Estrogen receptors, antiestrogens, and non-small cell lung cancer. Biochemistry (Mosc) 2010;75:1421-7.

5. Rohlff C, Blagosklonny MV, Kyle E, et al. Prostate cancer cell growth inhibition by tamoxifen is associated with inhibition of protein kinase $\mathrm{C}$ and induction of p21(wafl/cip1). Prostate 1998;37:51-9.

6. Cheng AL, Chuang SE, Fine RL, et al. Inhibition of the membrane translocation and activation of protein kinase $\mathrm{C}$, and potentiation of doxorubicin-induced apoptosis of hepatocellular carcinoma cells by tamoxifen. Biochem Pharmacol 1998;55:523-31.

7. Sharif TR, Sharif M. A novel approach for examining the anti-proliferative effect of protein kinase $\mathrm{C}$ inhibitors against human astrocytoma cells. Int J Oncol 1998;13:685-92.

8. Chen TC, Su S, Fry D, Liebes L. Combination therapy with irinotecan and protein kinase $\mathrm{C}$ inhibitors in malignant glioma. Cancer 2003;97:2363-73.

9. Lavie Y, Zhang ZC, Cao HT, et al. Tamoxifen induces selective membrane association of protein kinase C epsilon in MCF-7 human breast cancer cells. Int J Cancer 1998;77:928-32.
10. Wang XY, Wang Y, Liu HC. Tamoxifen lowers the MMP-9/TIMP-1 ratio and inhibits the invasion capacity of ER-positive non-small cell lung cancer cells. Biomed Pharmacother 2011;65:525-8.

11. Fang YJ, Pan ZZ, Li LR, et al. MMP7 expression regulated by endocrine therapy in ERbeta-positive colon cancer cells. J Exp Clin Cancer Res 2009;28:132.

12. Hoelting T, Siperstein AE, Duh QY, Clark OH. Tamoxifen inhibits growth, migration, and invasion of human follicular and papillary thyroid cancer cells in vitro and in vivo. J Clin Endocrinol Metab 1995;80:308-13.

13. Matsuoka H, Tsubaki M, Yamazoe Y, et al. Tamoxifen inhibits tumor cell invasion and metastasis in mouse melanoma through suppression of PKC/MEK/ERK and PKC/PI3K/Akt pathways. Exp Cell Res 2009;315:2022-32.

14. Xing RH, Mazar A, Henkin J, Rabbani SA. Prevention of breast cancer growth, invasion, and metastasis by antiestrogen tamoxifen alone or in combination with urokinase inhibitor B-428. Cancer Res 1997;57:3585-93.

15. Ahn SJ, Yoon MS, Hyuk S, et al. Phospholipase C-protein kinase C mediated phospholipase $\mathrm{D}$ activation pathway is involved in tamoxifen induced apoptosis. J Cell Biochem 2003;89:520-8.

16. Thiantanawat A, Long BJ, Brodie AM. Signaling pathways of apoptosis activated by aromatase inhibitors and antiestrogens. Cancer Res 2003;63:8037-50.

17. Feng Y, Huang J, Ding Y, et al. Tamoxifen-induced apoptosis of rat C6 glioma cells via PI3K/Akt, JNK and ERK activation. Oncol Rep 2010;24:1561-7.

18. Moodbidri MS, Shirsat NV. Activated JNK brings about accelerated apoptosis of Bcl-2-overexpressing C6 glioma cells on treatment with tamoxifen. J Neurochem 2005;92:1-9.

19. Zhang GJ, Kimijima I, Onda M, et al. Tamoxifen-induced apoptosis in breast cancer cells relates to down-regulation ofbcl-2, but not bax and bcl-X(L), without alteration of p53 protein levels. Clin Cancer Res 1999;5:2971-7.

20. Hawkin RA, Arends MJ, Ritchie AA, et al. Tamoxifen increases apoptosis but does not influence markers of proliferation in an MCF-7 xenograft model of breast cancer. Breast 2000;9:96-106.

21. Salami S, Karami-Tehrani F. Biochemical studies of apoptosis induced by tamoxifen in estrogen receptor positive and negative breast cancer cell lines. Clin Biochem 2003;36:247-53.

22. Han P, Kang JH, Li HL, et al. Antiproliferation and apoptosis induced by tamoxifen in human bile duct carcinoma QBC939 cells via upregulated p53 expression. Biochem Biophys Res Commun 2009;385:251-6.

23. Nazarewicz RR, Zenebe WJ, Parihar A, et al. Tamoxifen induces oxidative stress and mitochondrial apoptosis via stimulating mitochondrial nitric oxide synthase. Cancer Res 2007;67:1282-90.

24. Nagahara Y, Shiina I, Nakata K, et al. Induction of mitochondriainvolved apoptosis in estrogen receptor-negative cells by a novel tamoxifen derivative, ridaifen-B. Cancer Sci 2008;99:608-14.

25. Kallio A, Zheng A, Dahllund J, et al. Role of mitochondria in tamoxifeninduced rapid death of MCF-7 breast cancer cells. Apoptosis 2005;10:1395-410.

26. Bursch W, Ellinger A, Kienzl $\mathrm{H}$, et al. Active cell death induced by the anti-estrogens tamoxifen and ICI 164384 in human mammary carcinoma cells (MCF-7) in culture: the role of autophagy. Carcinogenesis 1996;17:1595-607.

27. Bursch W, Hochegger K, Torok L, et al. Autophagic and apoptotic types of programmed cell death exhibit different fates of cytoskeletal filaments. J Cell Sci 2000;113:1189-98.

28. Bilir A, Altinoz MA, Erkan M, et al. Autophagy and nuclear changes in FM3A breast tumor cells after epirubicin, medroxyprogesterone and tamoxifen treatment in vitro. Pathobiology 2001;69:120-6.

29. Scarlatti F, Bauvy C, Ventruti A, et al. Ceramide-mediated macroautophagy involves inhibition of protein kinase B and up-regulation of 
beclin 1. J Biol Chem 2004;279:18384-91.

30. Amaravadi RK, Yu D, Lum JJ, et al. Autophagy inhibition enhances therapy-induced apoptosis in a Myc-induced model of lymphoma. J Clin Invest 2007;11:326-36.

31. Nagarkatti N, Davis BA. Tamoxifen induces apoptosis in Fas+ tumor cells by upregulating the expression of Fas ligand. Cancer Chemother Pharmacol 2003;51:284-90.

32. Lochab S, Pal P, Kanaujiya JK, et al. Proteomic identification of E6AP as a molecular target of tamoxifen in MCF7 cells. Proteomics 2012;12:1363-77.

33. Lagadec C, Adriaenssens E, Toillon RA, et al. Tamoxifen and TRAIL synergistically induce apoptosis in breast cancer cells. Oncogene 2008;27:1472-7.

34. Zulehner N, Maurer M, Wesierska-Gadek J. Effect of anti-estrogen combined with roscovitine, a selective CDK inhibitor, on human breast cancer cells differing in expression of ER. J Exp Ther Oncol 2011;9:17-25.

35. W sierska-G dek J, Gritsch D, Zulehner N, et al. Roscovitine, a selective CDK inhibitor, reduces the basal and estrogen-induced phosphorylation of ER- $\alpha$ in human ER-positive breast cancer cells. J Cell Biochem 2011;112:761-72.

36. W sierska-G dek J, Gritsch D, Zulehner N, et al. Interference with ER$\alpha$ enhances the therapeutic efficacy of the selective CDK inhibitor roscovitine towards ER-positive breast cancer cells. J Cell Biochem 2011;112:1103-17.

37. O'Byrne KJ, Dalgleish AG, Browning MJ, et al. The relationship between angiogenesis and the immune response in carcinogenesis and the progression of malignant disease. Eur J Cancer 2000;36:15169.

38. Da Silva BB, da Silva Júnior RG, Borges US, et al. Quantification of angiogenesis induced in rabbit cornea by breast carcinoma of women treated with tamoxifen. J Surg Oncol 2005;90:77-80.

39. Blackwell KL, Haroon ZA, Shan S, et al. Tamoxifen inhibits angiogenesis in estrogen receptor-negative animal models. Clin Cancer Res 2000;6:4359-64.

40. Cáceres W, González S. Angiogenesis and cancer: recent advances. P R Health Sci J 2003;22:149-51.

41. Tong S, Chen Q, Shan SQ, et al. Quantitative comparison of the inhibitory effects of GW5638 and tamoxifen on angiogenesis in the cornea pocket assay. Angiogenesis 2006;9:53-8.

42. Gagliardi AR, Hennig B, Collins DC. Antiestrogens inhibit endothelial cell growth stimulated by angiogenic growth factors. Anticancer Res 1996;16:1101-6.

43. Butta A, MacLennan K, Flanders KC, et al. Induction of transforming growth factor beta 1 in human breast cancer in vivo following tamoxifen treatment. Cancer Res 1992;52:4261-4.

44. Garvin S, Dabrosin C. Tamoxifen inhibits secretion of vascular endothelial growth factor in breast cancer in vivo. Cancer Res 2003;63:8742-8.

45. Åberg UW, Saarinen N, Abrahamsson A, et al. Tamoxifen and flaxseed alter angiogenesis regulators in normal human breast tissue in vivo. PLoS One 2011;6:e25720.

46. McNamara DA, Harmey J, Wang JH, et al. Tamoxifen inhibits endothelial cell proliferation and attenuates VEGF-mediated angiogenesis and migration in vivo. Eur J Surg Oncol 2001;27:714-8.

47. Nilsson UW, Dabrosin C. Estradiol and tamoxifen regulate endostatin generation via matrix metalloproteinase activity in breast cancer in vivo. Cancer Res 2006;66:4789-94.

48. Nilsson UW, Garvin S, Dabrosin C. MMP-2 and MMP-9 activity is regulated by estradiol and tamoxifen in cultured human breast cancer cells. Breast Cancer Res Treat 2007;102:253-61.

49. Lindahl G, Saarinen N, Abrahamsson A, Dabrosin C. Tamoxifen, flaxseed, and the lignan enterolactone increase stroma- and cancer cell-derived IL-1Ra and decrease tumor angiogenesis in estrogen- dependent breast cancer. Cancer Res 2011;71:51-60.

50. Hu GF, Riordan JF, Vallee BL. A putative angiogenin receptor in angiogenin-responsive human endothelial cells. Proc Natl Acad Sci U S A 1997;94:2204-9.

51. Hu G, Riordan JF, Vallee BL. Angiogenin promotes invasiveness of cultured endothelial cells by stimulation of cell-associated proteolytic activities. Proc Natl Acad Sci U S A 1994;91:12096-100.

52. Jimi S, Ito K, Kohno K, Ono M, et al. Modulation by bovine angiogenin of tubular morphogenesis and expression of plasminogen activator in bovine endothelial cells. Biochem Biophys Res Commun 1995;211:476-83.

53. Miyake H, Hara I, Yamanaka K, et al. Increased angiogenin expression in the tumor tissue and serum of urothelial carcinoma patients is related to disease progression and recurrence. Cancer 1999;86:316-24

54. Shimoyama S, Kaminishi M. Increased angiogenin expression in gastric cancer correlated with cancer progression. J Cancer Res Clin Oncol 2000;126:468-74.

55. Shimoyama S, Yamasaki K, Kawahara M, Kaminishi M. Increased serum angiogenin concentration in colorectal cancer is correlated with cancer progression. Clin Cancer Res 1999;5:1125-30.

56. Shimoyama S, Gansauge F, Gansauge S, et al. Increased angiogenin expression in pancreatic cancer is related to cancer aggressiveness. Cancer Res 1996;56:2703-6.

57. Olson KA, Byers HR, Key ME, Fett JW. Prevention of human prostate tumor metastasis in athymic mice by antisense targeting of human angiogenin. Clin Cancer Res 2001;7:3598-605.

58. Sheen-Chen SM, Eng HL, Chen WJ, et al. Serum level of angiogenin in breast cancer. Anticancer Res 2000;20:4769-71.

59. Montero S, Guzmán C, Cortés-Funes H, Colomer R. Angiogenin expression and prognosis in primary breast carcinoma. Clin Cancer Res 1998;4:2161-8.

60. Eppenberger U, Kueng W, Schlaeppi JM, et al. Markers of tumor angiogenesis and proteolysis independently define high- and low-risk subsets of node-negative breast cancer patients. J Clin Oncol 1998;16:3129-36.

61. Campo L, Turley H, Han C, Pezzella F, et al. Angiogenin is up-regulated in the nucleus and cytoplasm in human primary breast carcinoma and is associated with markers of hypoxia but not survival. J Pathol 2005;205:585-91.

62. Nilsson UW, Abrahamsson A, Dabrosin C. Angiogenin regulation by estradiol in breast tissue: tamoxifen inhibits angiogenin nuclear translocation and antiangiogenin therapy reduces breast cancer growth in vivo. Clin Cancer Res 2010;16:3659-69.

63. Hotta T, Tanimura H, Yamaue H. Tamoxifen circumvents the multidrug resistance in fresh human gastrointestinal cancer cells. J Surg Res 1996;66:31-5.

64. Shen LZ, Hua YB, Yu XM. Tamoxifen can reverse multidrug resistance of colorectal carcinoma in vivo. World J Gastroenterol 2005;11:1060-4.

65. Safa AR, Roberts S, Agresti M, Fine RL. Tamoxifen aziridine, a novel affinity probe for P-glycoprotein in multidrug resistant cells. Biochem Biophys Res Commun 1994;202:606-12.

66. Rao US, Fine RL, Scarborough GA. Antiestrogens and steroid hormones: substrates of the human P-glycoprotein. Biochem Pharmacol 1994;48:287-92.

67. Liu ZH, Ma YL, He YP, et al. Tamoxifen reverses the multi-drug-resistance of an established human cholangiocarcinoma cell line in combined chemotherapeutics. Mol Biol Rep 2011;38:1769-75.

68. Bogush EA, Ravcheeva AB, Bogush TA, et al. A new marker of tamoxifen resistance of estrogen receptor-positive breast cancer. Dok Biochem Biophys 2007;413:83-7.

69. Bogush TA, Dudko EA, Bogush EA, et al. MRP as a new predictive marker of tamoxifen efficiency in treatment of estrogen receptor-positive breast cancer. Dokl Biochem Biophys 2010;430:36-40. 